Copyright by the American Institute of Physics (AIP). Dong, SX; Li, JF; Viehland, D., "Ultrahigh magnetic field sensitivity

in laminates of TERFENOL-D and Pb(Mg1/3Nb2/3) O-3-PbTiO3 crystals," Appl. Phys. Lett. 83, 2265 (2003); http:// dx.doi.org/10.1063/1.1611276

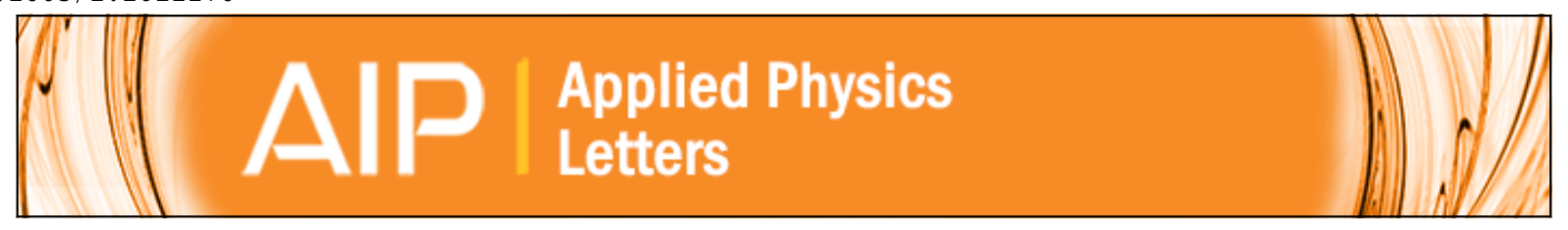

Ultrahigh magnetic field sensitivity in laminates of TERFENOL-D and $\mathrm{Pb}$ ( $\mathrm{Mg} 1 / 3 \mathrm{Nb} 2 / 3$ ) 03 - PbTiO 3 crystals

Shuxiang Dong, Jie-Fang Li, and D. Viehland

Citation: Applied Physics Letters 83, 2265 (2003); doi: 10.1063/1.1611276

View online: http://dx.doi.org/10.1063/1.1611276

View Table of Contents: http://scitation.aip.org/content/aip/journal/apl/83/11?ver=pdfcov

Published by the AIP Publishing

Over 700 papers \&

presentations on

multiphysics simulation vis now

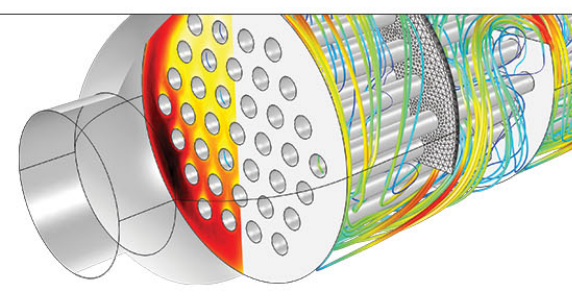




\title{
Ultrahigh magnetic field sensitivity in laminates of TERFENOL-D and $\mathrm{Pb}\left(\mathrm{Mg}_{1 / 3} \mathrm{Nb}_{2 / 3}\right) \mathrm{O}_{3}-\mathrm{PbTiO}_{3}$ crystals
}

\author{
Shuxiang Dong, ${ }^{\text {a) }}$ Jie-Fang Li, and D. Viehland \\ Materials Science and Engineering, Virginia Tech, Blacksburg, Virginia 24061
}

(Received 29 April 2003; accepted 21 July 2003)

\begin{abstract}
It has been found that laminate composites of longitudinally magnetized magnetostrictive TERFENOL-D and a transversely poled piezoelectric $\mathrm{Pb}\left(\mathrm{Mg}_{1 / 3} \mathrm{Nb}_{2 / 3}\right) \mathrm{O}_{3}-\mathrm{PbTiO}_{3}$ crystal have extremely high magnetic field sensitivity. At room temperature, an output voltage with an exceptionally good linear response to an ac magnetic field $H_{\mathrm{ac}}$ was found over the range of $10^{-11}<H_{\mathrm{ac}}<10^{-3}$ T. (C) 2003 American Institute of Physics. [DOI: 10.1063/1.1611276]
\end{abstract}

The magnetoelectric (ME) effect is a polarization $P$ response to an applied magnetic field $H .^{1}$ ME effects have been reported in single phase materials; ${ }^{2-4}$ however, to date, a ME material with significant coupling has not been reported. It is known that piezoelectric/magnetostrictive composites have better ME properties, ${ }^{2-17}$ than single phase materials. Most previous investigations have focused on laminates whose piezoelectric/magnetostrictive layers are polarized/magnetized in their thickness/transverse directions, respectively.

In this investigation, magnetoelectric composites were made from a $\langle 001\rangle$-oriented $0.7 \mathrm{~Pb}\left(\mathrm{Mg}_{1 / 3} \mathrm{Nb}_{2 / 3}\right) \mathrm{O}_{3}-0.3 \mathrm{PbTiO}_{3} \quad(\mathrm{PMN}-\mathrm{PT})$ piezoelectric single crystal laminated between two TERFENOL-D $\left(\mathrm{Tb}_{1-x} \mathrm{Dy}_{x} \mathrm{Fe}_{2-y},\right)$ layers, as shown in Fig. 1. The length and width of each layer was 12 and $6 \mathrm{~mm}$, respectively. The total laminate cross-sectional area $A$ was $6 \times 2.5 \mathrm{~mm}^{2}$. This miniature long-type configuration consists of TERFENOL-D layers magnetized in their length or longitudinal direction and a piezoelectric layer poled in its thickness or transverse direction-a $L-T$ laminate composite. The configuration intensifies the principle strain/vibration along the longitudinal axis. Correspondingly, this favors the tensor property matrix for TERFENOL-D, as its longitudinal strain is much higher than the transverse. Our configuration is significantly different than previous reports. ${ }^{5-15,17}$

In our $L-T$ laminate, because the piezomagnetic and piezoelectric layers are mutually coupled via strain $S(z)$ and stress $T(z)$, application of $H$ along the length axis of the magnetostrictive layer puts the piezoelectric one into forced oscillation along this direction. A voltage is generated across the thickness or transverse direction of the piezoelectric layer, via the transverse piezoelectric constant $d_{31, p}$. Prior analysis of magnetoestrictive/piezoelectric laminates has simply combined the magnetostrictive and piezoelectric constitutive equations. ${ }^{7,9,12,15-17}$ This is inadequate to understand energy coupling between layers under dynamic drive. In our analysis, we used an equation of motion to couple the two constitutive equations. By applying Newton's second law of motion to the laminate and finding analogous electrical parameters, ${ }^{18,19}$ the induced ME voltage as a function of ap-

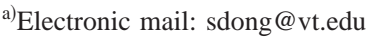

plied ac magnetic field $H_{\text {ac }}\left(f \ll f_{0}\right.$, where $f_{0}$ is the resonance frequency of the laminate) for the $L-T$ laminate is

$$
V_{\mathrm{ME}}^{L, T}=\frac{n(1-n) A d_{33, m} d_{31, p}^{2}}{\varepsilon_{33}^{T} s_{11}^{E}\left[n s_{11}^{E}\left(1-k_{31, p}^{2}\right)+(1-n) s_{33}^{H}\right]} H_{\mathrm{ac}} ;
$$

where $s_{11}^{E}$ and $s_{33}^{H}$ are the elastic compliances of the piezoelectric and magnetostrictive layers respectively, $k_{31, p}$ and $\varepsilon_{33}^{T}$ are electromechanical coupling coefficient and dielectric constant at constant stress of the piezoelectric material, and $d_{33, m}$ and $d_{31, p}$ are the longitudinal piezomagnetic and transverse piezoelectric coefficients, respectively. This equation predicts that $V_{\mathrm{ME}}^{L, T}$ should be a linear function of $H_{\mathrm{ac}}$. Using the materials parameters for PMN-PT and TERFENOL-D in Table I, the maximum value of $V_{\mathrm{ME}}^{L, T}$ can be estimated as $123.7 \mathrm{mV}$ at $H_{\mathrm{ac}}=1 \mathrm{Oe}$, assuming a thickness ratio $n$ $=0.50$ for the TERFENOL-D layers. In addition, the equivalent circuit model predicts an optimum thickness ratio $n_{\text {optim }}$ for the TERFENOL-D layers of

$$
n_{\text {optim }}=\frac{1}{1+\sqrt{\alpha}} \text {, where } \alpha=\left(1-k_{31, p}^{2}\right) \frac{s_{11}^{E}}{s_{33}^{H}} .
$$

Using typical materials parameters listed in Table I, ${ }^{18,20}$ we estimate $n_{\text {optim }} \sim 0.50$. This is the value of the thickness ratio $n$ used in our laminate design.

The voltage induced across the PMN-PT layer of a laminate prototype was measured for various $H_{\mathrm{dc}}$ (dc magnetic biases) and $H_{\mathrm{ac}}$ using a lock-in amplifier method. A pair of Helmholtz coils was used to generate a small $H_{\mathrm{ac}}$, via an input current $I_{\text {coil }}$. The induced ME voltage of the $L-T$ laminate was found to be a linear function of $H_{\mathrm{ac}}$, as shown in Fig. 2 and as predicted by Eq. (1). The ME voltage was

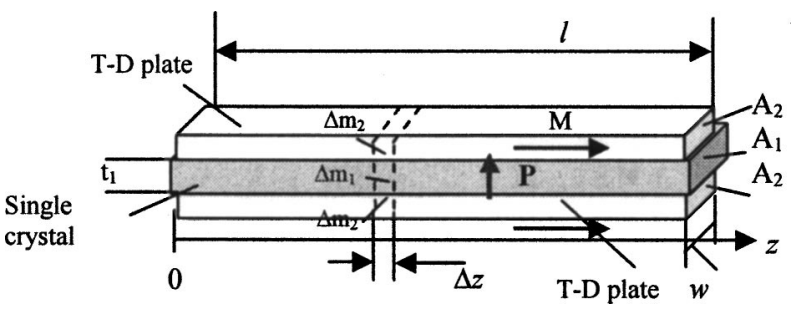

FIG. 1. Long-type configuration of our $L-T$ mode magnetostrictive/ piezoelectric laminate composite. $\Delta m_{1}$ and $\Delta m_{2}$ are PMN-PT single crystal mass unit and TERFENOL-D mass unit at axial $z$. The arrow $M$ and $P$ represent the magnetization and polarization directions, respectively. 
TABLE I. Electromechanical and magnetoelastic materials parameters for TERFENOL-D and $\langle 001\rangle$-oriented PMN-PT crystals.

\begin{tabular}{|c|c|c|c|c|c|c|c|}
\hline & $d_{33, m}$ or $d_{33, p}$ & $d_{31, m}$ or $d_{31, p}$ & $s_{11}^{H}$ or $s_{11}^{E}$ & $s_{33}^{H}$ or $s_{33}^{E}$ & $k_{33}$ & $k_{31}$ & $\varepsilon_{33 \mathrm{effec}}^{T} \mathrm{c}$ \\
\hline $\begin{array}{l}\text { TERFENOL-D } \\
\langle 001\rangle^{\mathrm{b}} \\
\text { PMN-PT }\end{array}$ & $\begin{array}{l}1.2 \times 10^{-8} \mathrm{~Wb} / \mathrm{N} \\
2820 \mathrm{pC} / \mathrm{N}\end{array}$ & $\begin{array}{l}-5.8 \times 10^{-9} \mathrm{~Wb} / \mathrm{N} \\
-1330 \mathrm{pC} / \mathrm{N}\end{array}$ & $\begin{array}{l}125 \times 10^{-12} \mathrm{~m}^{2} / \mathrm{N} \\
69.0 \times 10^{-12} \mathrm{~m}^{2} / \mathrm{N}\end{array}$ & $\begin{array}{l}40 \times 10^{-12} \mathrm{~m}^{2} / \mathrm{N} \\
119 \times 10^{-12} \mathrm{~m}^{2} / \mathrm{N}\end{array}$ & $\begin{array}{l}0.7 \\
0.95\end{array}$ & 0.58 & 2134 \\
\hline
\end{tabular}

${ }^{\mathrm{a} C i t e d ~ f r o m ~ R e f . ~} 18$.

${ }^{\mathrm{b}}$ Cited from Ref. 20.

${ }^{\mathrm{c}}$ Measured value after assembling.

also found to depend on $H_{\mathrm{dc}}$, reflecting the dc-biased piezomagnetic behavior of TERFENOL- $\mathrm{D}^{12,18,21}$ which is maximum near the inflection point of the quadratic strainmagnetic field $(\varepsilon-H)$ curve. The maximum ME voltage using a drive of $H_{\mathrm{ac}}=1 \mathrm{Oe}$ was $\sim 110 \mathrm{mV}$ under a $H_{\mathrm{dc}}$ $=400 \mathrm{Oe}$. This is consistent with the value predicted above, and translates into a maximum ME field coefficient for the $L-T$ laminate of $|d E / d H|_{L-T}=2.2 \mathrm{~V} / \mathrm{cm} \mathrm{Oe}$, at $\left(H_{\mathrm{dc}}\right.$ $=400 \mathrm{Oe}$ ).

The ME voltage was then measured over a broad magnetic field range of $10^{-11}<x<10^{-3} \mathrm{~T}$, as shown in Fig. 3 . In this figure, the induced $\mathrm{ME}$ voltage can be seen to have an excellent linear response to the amplitude of $H_{\mathrm{ac}}$ over $\sim 8$ orders of magnitude. These measurements were performed at ambient conditions, without magnetic shielding. The results unambiguously demonstrate that the $L-T$ laminate has an ultrahigh sensitivity to small magnetic field variations. The limit of sensitivity of the laminate was then determined. Figure 3 shows the induced voltage and phase signals using an extremely low input current into the Helmoltz coils of $I_{\text {coil }}$ $=22 \mathrm{nA}$. The field generated by the coils was $H_{\mathrm{ac}}=6.1$ $\times 10^{-11} \mathrm{~T}(\mathrm{rms})$. The induced ME voltage under this low $H_{\text {ac }}$ was $\sim 45 \mathrm{nV}$ (rms). The system noise was determined to be $\sim 20 \mathrm{nV}$. Thus, the limit of sensitivity of the laminate to small magnetic field variations is $\sim 4 \times 10^{-11} \mathrm{~T}$, which corresponds to a noise-equivalent ME voltage of $30 \mathrm{nV}(\mathrm{rms})$. This noise floor can be significantly lowered by careful shielding. With our circuitry or a null circuit, it is feasible to detect small voltage changes of $\sim 1 \mathrm{nV}$. Thus, our laminate has the potential to detect magnetic field variations in the picotesla range and below.

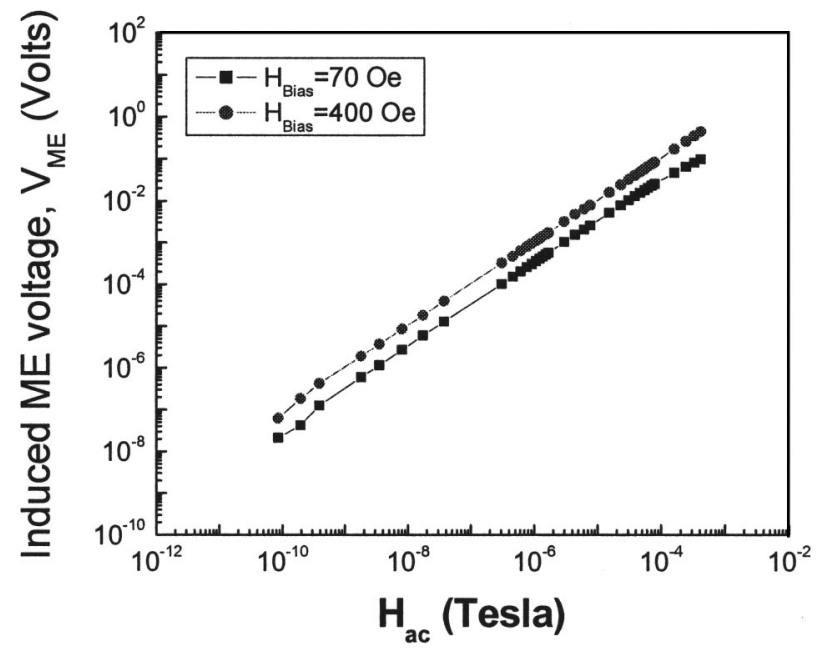

FIG. 2. Induced ME voltage as a function of magnetic field from $10^{-11}$ $<H_{\mathrm{ac}}<10^{-3} \mathrm{~T}$.
Our miniature long-type laminate of TERFENOL-D and $\mathrm{Pb}\left(\mathrm{Mg}_{1 / 3} \mathrm{Nb}_{2 / 3}\right) \mathrm{O}_{3}-\mathrm{PbTiO}_{3}$, which is driven in a $L-T$ mode, has extremely high magnetic field sensitivity. The results demonstrate a magnetic field sensitivity limit of significantly $<4 \times 10^{-11} \mathrm{~T}$. This represents an enhancement in sensitivity limitation of nearly $10^{4} \times$, over previous single phase or two-phase laminate composites (for example, see Ref. 21). This is a significant achievement for a totally passive $(\sim 0 \mu \mathrm{W}$ power consumed) and compact magnetic field sensor that has an output voltage linearly proportional to $H$. It offers opportunities in sensitive low-level magnetic field and/or electric current sensing. For example, the most sensitive magnetic field sensors are superconducting quantum interference devices (SQUIDs), which have a maximum sensitivity limit of $\approx 10^{-14} \mathrm{~T}$, when operated below $77 \mathrm{~K}^{22,23}$ The next best methods for ultralow magnetic field detection are optical pumping and nuclear precision, ${ }^{22}$ offering sensitivity limits of $\approx 10^{-11} \mathrm{~T}$.

In summary, an ultrahigh magnetic field sensitivity has been found in a miniature long-type composite of a $\langle 001\rangle$ oriented PMN-PT crystal laminated between two TERFENOL-D layers that is operated in a $L-T$ mode. The results demonstrate: (i) a giant $\mathrm{ME}$ voltage of $>110 \mathrm{mV}$ for $H_{\mathrm{ac}}=1 \mathrm{Oe}$; (ii) an extremely high magnetic field sensitivity of $<4 \times 10^{-11} \mathrm{~T}$ which has significant potential for picotesla resolution; and (iii) an exceptional linear ME voltage response to changes in magnetic field over a range of $10^{-11}$ $<H_{\mathrm{ac}}<10^{-3} \mathrm{~T}$.

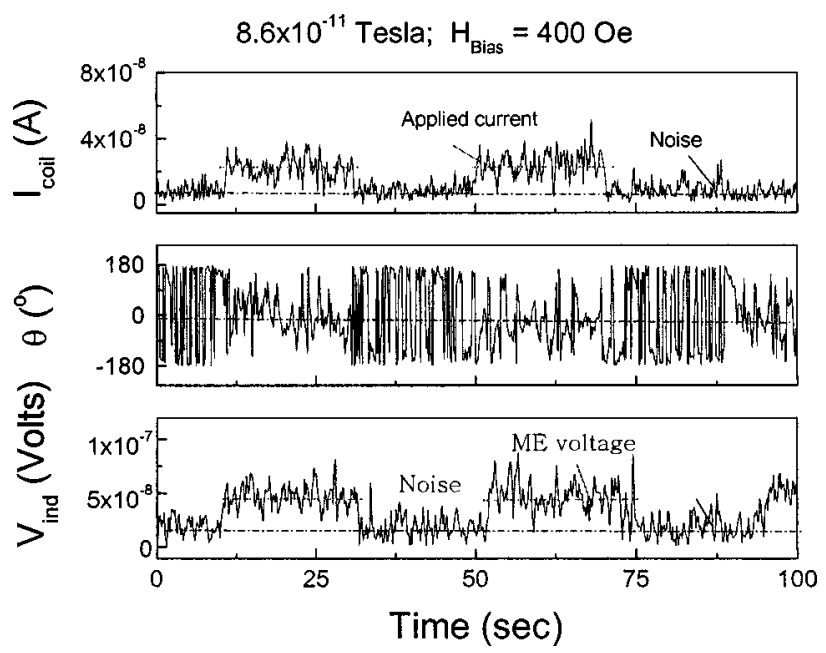

FIG. 3. Determination of sensitivity limit of laminate. (a) Input current into Helmholtz $I_{\text {coil }}$ as a function of time, (b) phase response $\theta$ of laminate, and (c) voltage response of laminate. Due to the low coil current, the applied magnetic field was $H_{\text {ac }}=6 \times 10^{-11} \mathrm{~T}$ (rms). Measurements werc performed under a magnetic bias of $H_{\mathrm{dc}}=400 \mathrm{Oe}$. 
This research was supported by the Office of Naval Research under Grant Nos. N000140210340, N000140210126, and MURI N000140110761.

${ }^{1}$ L. D. Landau and E. Lifshitz, Electrodynamics of Continuous Media (Pergamon, Oxford, 1960), p. 119.

${ }^{2}$ S. Di Matteo and A. G. M. Jansen, Phys. Rev. B 66, 100402 (2002).

${ }^{3}$ I. E. Dzyaloshinskii, Sov. Phys. JETP 37, 628 (1960).

${ }^{4}$ L. Wiegelmann, A. A. Stepanov, I. M. Vitebsky, A. G. M. Jansen, and P. Wyder, Phys. Rev. B 49, 10039 (1994).

${ }^{5}$ J. Ryu, A. V. Carazo, K. Uchino, and H. Kim, J. Electroceram. 7, 24 (2001).

${ }^{6}$ T. Wu and J. Huang, Int. J. Solids Struct. 37, 3009 (2000).

${ }^{7}$ C. W. Nan, M. Li, and J. H. Huang, Phys. Rev. B 63, 144415 (2001).

${ }^{8}$ C. W. Nan, L. Liu, N. Cai, J. Zhai, Y. Ye, and Y. H. Lin, Appl. Phys. Lett. 81, 3831 (2002).

${ }^{9}$ M. Avellaneda and G. Harshe, J. Intell. Mater. Syst. Struct. 5, 501 (1994).

${ }^{10}$ J. Ryu, A. Vazquez Carazo, K. Uchino, and H. Kim, Jpn. J. Appl. Phys., Part 1 40, 4948 (2001).

${ }^{11}$ G. Srinivasan, E. Rasmussen, B. Levin, and R. Hayes, Phys. Rev. B 65, 134402 (2002).
${ }^{12}$ G. Srinivasan, E. Rasmussen, J. Gallegos, R. Srinivasan, Y. Bokhan, and V. Laletin, Phys. Rev. B 64, 214408 (2001).

${ }^{13}$ J. Ryu, S. Priya, K. Uchino, H. E. Kim, and D. Viehland, J. Korean Ceramic Society 39, 813 (2002).

${ }^{14}$ G. Srinivasan, V. M. Laletin, R. Hayes, N. Puddubnaya, E. T. Rasmussen, and D. J. Fekel, Solid State Commun. 124, 373 (2002).

${ }^{15}$ M. I. Bichurin, V. M. Petrov, and G. Srinivasan, J. Appl. Phys. 92, 7681 (2002).

${ }^{16}$ K. Mori and M. Wuttig, Appl. Phys. Lett. 81, 100 (2002).

${ }^{17}$ W. Mason, Physical Acoustics, Principle and Methods, p. 263

${ }^{18}$ G. Engdahl, Magnetostrictive Materials Handbook (Academic, New York, 2000).

${ }^{19}$ T. Ikeda, Fundamentals of Piezoelectricity (Oxford University Press, New York, 1990).

${ }^{20}$ R. Zhang, B. Jiang, and W. Cao, J. Appl. Phys. 90, 3471 (2001).

${ }^{21} \mathrm{Y}$. Li, 17, 10 (2000) (sensors online: http://www.sensorsmag.com/articles/ 1000/52/index.htm).

${ }^{22}$ J. Clarke and R. H. Koch, Science (Washington, DC, U.S.) 242, 217 (1988).

${ }^{23}$ J. Lenz, Proc. IEEE 78, 973 (1990). 